\title{
B-Meson Wavefunction in the Wandzura-Wilczek Approximation
}

\author{
Tao, Huang ${ }^{1,2 *}$, Xing-Gang, $\mathrm{Wu}^{1 \dagger}$ and Ming-Zhen, Zhou ${ }^{1 \ddagger}$ \\ ${ }^{1}$ CCAST(World Laboratory), P.O.Box 8730, Beijing 100080, P.R.China, \\ ${ }^{2}$ Institute of High Energy Physics, Chinese Academy of Sciences, \\ P.O.Box 918(4), Beijing 100049, P.R. China.
}

\begin{abstract}
The B-meson wavefunction has been studied with the help of the vacuum-to-meson matrix element of the nonlocal operators in the heavy quark effective theory. In order to obtain the Wandzura-Wilczek-type B-meson wavefunction, we solve the equations which are derived from the equation of motion of the light spectator quark in the B meson by using two different assumptions. Under the condition that $\omega_{0}=2 \bar{\Lambda}$, the solutions for the B-meson wavefunction in this paper agree well with the one obtained by directly taking the heavy quark limit $m_{b} \rightarrow \infty$. Our results show that the equation of motion of the light spectator quark in the B meson can impose a strong constraint on the B-meson wavefunctions $\Psi_{ \pm}\left(\omega, z^{2}\right)$. Based on the obtained results, we claim that both its distribution amplitudes $\phi_{B}(\omega)$ and $\bar{\phi}_{B}(\omega)$ are important for calculating the B meson decays.
\end{abstract}

PACS numbers: 12.38.Aw, 12.39.Hg, 14.40.Nd

Keywords: B meson, wavefunction, heavy quark limit.

\footnotetext{
* email: huangtao@mail.ihep.ac.cn

$\dagger$ email: wuxg@mail.ihep.ac.cn

$\ddagger$ email: zhoumz@mail.ihep.ac.cn
} 


\section{INTRODUCTION}

Along with the theoretical and experimental progresses, B physics is attracting more and more attentions. The non-perturbative light-cone (LC) wavefunction/distribtuion amplitude (DA) of the $\mathrm{B}$ meson plays an important role in making reliable predictions for exclusive $\mathrm{B}$

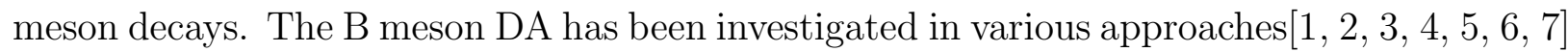
and is the basis of the collinear factorization [1]. Ref. 2] shows that the B meson DA is not normalizable. Such feature of the B meson DA does not cause a problem in practice [4], but it does introduce an ambiguity in defining the B meson decay constant $f_{B}$. Recently, Ref. [8] claims that it is the $\mathrm{B}$ meson wavefunction that is more relevant to the $\mathrm{B}$ decays and in the framework of the $k_{T}$-factorization theorem [9], they proved that the B meson wavefunction is renormalizable after taking into account renormalization-group evolution effects. In the $k_{T^{-}}$factorization theorem, by taking into account the transverse momentum dependence $\left(k_{T^{-}}\right.$ dependence) into the non-perturbative wavefunctions and the hard scattering, the endpoint singularity (an example can be found in Ref.[10]) coming from the collinear factorization can be cured. Theoretically, it is an important issue to study the longitudinal and transverse momentum dependence of the B wavefunction, since it provides a major source of uncertainty in the calculations of the $\mathrm{B}$ decays.

Ref. 11] presents an analytic solution for the B-meson wavefunction, which satisfies the constraints coming from the equations of motion and the heavy-quark symmetry [12]. They find that the "Wandzura-Wilczek-type" contribution 13] (WW approximation), which corresponds to the valence quark distribution, can be determined uniquely in analytic form in terms of the "effective mass" $(\bar{\Lambda})$ of the meson state, which is defined in the Heavy Quark Effective Theory (HQET) 14]. However, in Ref.[11], two extra constraints for the B-meson wavefunction come from the heavy quark limit, $m_{b} \rightarrow \infty$. Since the mass of b-quark is limited, such condition might be too strong, and we will not take such limit in our present calculation. In the following, we shall solve the two equations that are derived from the equation of motion of the light spectator quark on the basis of some physical considerations. 


\section{EQUATIONS UNDER THE WW APPROXIMATION}

In HQET 14], the wavefunctions $\tilde{\Psi}_{ \pm}\left(t, z^{2}\right)$ of the B meson can be defined in terms of the vacuum-to-meson matrix element of the nonlocal operators:

$$
\left\langle 0\left|\bar{q}(z) \Gamma h_{v}(0)\right| \bar{B}(p)\right\rangle=-\frac{i f_{B} M}{2} \operatorname{Tr}\left[\gamma_{5} \Gamma \frac{1+\psi}{2} \times\left\{\tilde{\Psi}_{+}\left(t, z^{2}\right)-\not \frac{\tilde{\Psi}_{+}\left(t, z^{2}\right)-\tilde{\Psi}_{-}\left(t, z^{2}\right)}{2 t}\right\}\right] .
$$

Here, $z^{\mu}=\left(0, z^{-}, \mathbf{z}_{\perp}\right), z^{2}=-\mathbf{z}_{\perp}^{2}, v^{2}=1, t=v \cdot z$, and $p^{\mu}=M v^{\mu}$ is the 4-momentum of the B meson with mass $M . h_{v}(x)$ denotes the effective $b$-quark field. $\Gamma$ is a generic Dirac matrix. The path-ordered gauge factors are implied in between the constituent fields. Note that in the above definition, the separation between the quark and the antiquark is not restricted on the $\mathrm{LC}\left(z^{2}=0\right)$.

The effective mass $(\bar{\Lambda})$ is much smaller than the B meson mass, so the light spectator quark in the B meson can be treated as on mass shell with high precision. Based on the QCD equation of motion for the light spectator quark, we can obtain a set of equations for $\tilde{\Psi}_{ \pm}\left(t, z^{2}\right)$ under the WW approximation, i.e.

$$
\frac{\partial \tilde{\Psi}_{-}\left(t, z^{2}\right)}{\partial t}-\frac{\tilde{\Psi}_{+}\left(t, z^{2}\right)-\tilde{\Psi}_{-}\left(t, z^{2}\right)}{t}-\frac{z^{2}}{t} \frac{\partial}{\partial z^{2}}\left[\tilde{\Psi}_{+}\left(t, z^{2}\right)-\tilde{\Psi}_{-}\left(t, z^{2}\right)\right]=0,
$$

and

$$
\frac{\partial \tilde{\Psi}_{+}\left(t, z^{2}\right)}{\partial t}-\frac{\partial \tilde{\Psi}_{-}\left(t, z^{2}\right)}{\partial t}-\frac{\tilde{\Psi}_{+}\left(t, z^{2}\right)-\tilde{\Psi}_{-}\left(t, z^{2}\right)}{t}+4 t \frac{\partial \tilde{\Psi}_{+}\left(\omega, z^{2}\right)}{\partial z^{2}}=0 .
$$

When taking the LC limit $z^{2} \rightarrow 0$, the above two equations agree well with the ones in

Refs. [6, 7]. By doing the Fourier transformation, $\tilde{\Psi}_{ \pm}\left(t, z^{2}\right)=\int d \omega e^{-i \omega t} \Psi_{ \pm}\left(\omega, z^{2}\right)$, Eqs.(23) become that

$$
\omega \frac{\partial \Psi_{-}\left(\omega, z^{2}\right)}{\partial \omega}+z^{2}\left(\frac{\partial \Psi_{+}\left(\omega, z^{2}\right)}{\partial z^{2}}-\frac{\partial \Psi_{-}\left(\omega, z^{2}\right)}{\partial z^{2}}\right)+\Psi_{+}\left(\omega, z^{2}\right)=0,
$$

and

$$
\left(\omega \frac{\partial}{\partial \omega}+2\right)\left[\Psi_{+}\left(\omega, z^{2}\right)-\Psi_{-}\left(\omega, z^{2}\right)\right]+4 \frac{\partial^{3} \Psi_{+}\left(\omega, z^{2}\right)}{\partial \omega^{2} \partial z^{2}}=0 .
$$

Here, $\omega v^{+}$has the meaning of the LC projection $k^{+}$of the light-antiquark momentum in the B meson. The exact solution of Eqs. (445) will give strong constraints on the longitudinal and transverse momentum dependence of the B wavefunction under the WW approximation. 


\section{SOLUTIONS FOR THE B WAVEFUNCTION}

At present, one can not obtain the exact solution for the B-meson wavefunctions $\Psi_{ \pm}\left(\omega, z^{2}\right)$ only with Eqs.(45). Some prescriptions must be made. Under the prescription that $\Psi_{ \pm}\left(\omega, z^{2}\right)$ have the same $z^{2}$-dependence and by taking the heavy quark limit $m_{b} \rightarrow \infty$, Ref.[11] obtained an analytic solution for $\Psi_{ \pm}\left(\omega, z^{2}\right)$. In the present paper, we do not take such limit and try to solve Eqs.(45) by taking two other prescriptions.

Before solving Eqs.(45), we define two functions $\phi_{ \pm}(\omega)$ in the following way,

$$
\begin{aligned}
\phi_{ \pm}(\omega) & \equiv \lim _{z^{2} \rightarrow 0} \Psi_{ \pm}\left(\omega, z^{2}\right) \\
& =\left.\int d^{2} \mathbf{k}_{\perp} \exp \left(i \mathbf{k}_{\perp} \cdot \mathbf{z}_{\perp}\right) \tilde{\Psi}_{ \pm}\left(\omega, \mathbf{k}_{\perp}^{2}\right)\right|_{\mathbf{z}_{\perp} \rightarrow 0}=\int d^{2} \mathbf{k}_{\perp} \tilde{\Psi}_{ \pm}\left(\omega, \mathbf{k}_{\perp}\right)
\end{aligned}
$$

where the second line shows that $\phi_{ \pm}(\omega)$ are precisely the B meson DAs. Taking the LC limit $\left(z^{2} \rightarrow 0\right)$ in Eq.(4), one can directly obtain a relation between the two DAs $\phi_{+}(\omega)$ and $\phi_{-}(\omega)$ of the B meson,

$$
\omega \frac{\partial \phi_{-}(\omega)}{\partial \omega}+\phi_{+}(\omega)=0
$$

which agrees with the one obtained in Refs. [6], 7].

Furthermore, one can prove that if the B-meson wavefunctions $\Psi_{ \pm}\left(\omega, z^{2}\right)$ can be constructed in the following way,

$$
\Psi_{ \pm}\left(\omega, z^{2}\right)=\phi_{ \pm}(\omega) \cdot \chi\left(\omega, z^{2}\right)
$$

then $\chi\left(\omega, z^{2}\right)$ must be a function of the correlated variable $\left[z^{2} \omega\left(\omega_{0}-\omega\right)\right]^{1}$, where $\omega_{0}$ is the maximum value of $\omega$. The ansatz (8) shows that the two wavefunctions $\Psi_{ \pm}\left(\omega, z^{2}\right)$ have the same $z^{2}$-dependence $\chi\left(\omega, z^{2}\right)$. A boundary condition for $\chi\left(\omega, z^{2}\right)$ can be derived from Eqs.(68), i.e. $\lim _{z^{2} \rightarrow 0} \chi\left(\omega, z^{2}\right)=1$. From the above ansatz, we can solve Eqs.(45) exactly without taking the heavy quark limit $m_{b} \rightarrow \infty$.

Substituting Eq.(8) into Eq.(41), we obtain

$$
\chi\left(\omega, z^{2}\right)=\xi\left(\omega \cdot \phi_{-}(\omega) \cdot z^{2}\right) \equiv \xi(y)
$$

\footnotetext{
${ }^{1}$ The special case that $\chi\left(\omega, z^{2}\right)$ has nothing to do with the variable $\omega$ has been discussed in Ref. 7] and will not be discussed here.
} 
where $\xi(y)$ is the function of a single variable $y=\left[\omega \cdot \phi_{-}(\omega) \cdot z^{2}\right]$. The explicit form of $\phi_{-}(\omega)$ can be derived from Eqs.(59), which reads

$$
\phi_{-}(\omega)=a_{1} \omega+a_{0}, \quad a_{1}=-\frac{\xi(0)}{4 \xi^{\prime}(0)}
$$

where the parameter $a_{0}$ is to be determined. With the help of the above results, Eq.(15) changes to

$$
\frac{y}{2}\left(\frac{\left(a_{0}+2 a_{1} \omega\right)^{2}}{\left(a_{0}+a_{1} \omega\right)\left(a_{0}+3 a_{1} \omega\right)}\right) \frac{d\left[a_{1} \sigma(y)+\left(1+a_{1}\right) \xi(y)\right]}{d y}+\left[a_{1} \sigma(y)+\left(1+a_{1}\right) \xi(y)\right]=0,
$$

with $\sigma(y)=4 y \xi^{\prime \prime}(y)+4 \xi^{\prime}(y)-\xi(y)$. Eq.(11) should be satisfied for all values of $\omega$, and then we have to set,

$$
a_{1} \sigma(y)+\left(1+a_{1}\right) \xi(y)=0
$$

The non-trivial solution of the above equation is

$$
\xi(y)=J_{0}\left(\sqrt{y / a_{1}}\right), \quad\left(a_{1}<0\right)
$$

where $J_{0}$ is the zero-th normal Bessel function. Here $a_{1}$ must be less than zero, because if $a_{1}=0$, then it will lead to $\phi_{+}(\omega) \equiv 0$; and if $a_{1}>0$, then one may obtain a self-contradictory result from Eq.(12): $a_{1}=-K_{0}(0) /\left(4 K_{0}^{\prime}(0)\right) \equiv 0$.

After doing the Fourier transformation for the transverse part, $\tilde{\Psi}_{ \pm}\left(\omega, \mathbf{k}_{\perp}\right)=$ $\int d^{2} \mathbf{z}_{\perp} \exp \left(-i \mathbf{k}_{\perp} \cdot \mathbf{z}_{\perp}\right) \Psi_{ \pm}\left(\omega, z^{2}\right) /(2 \pi)^{2}$, the final solution for the B-meson wavefunctions can be written as,

$$
\tilde{\Psi}_{ \pm}\left(\omega, \mathbf{k}_{\perp}\right)=\phi_{ \pm}(\omega) \frac{\delta\left(\mathbf{k}_{\perp}^{2}-\omega\left(\omega_{0}-\omega\right)\right)}{\pi}
$$

where $\omega_{0}=\left(-a_{0} / a_{1}\right)$ is the maximum value of $\omega$ and the normalized DAs take the form,

$$
\phi_{+}(\omega)=\frac{2}{\omega_{0}^{2}} \theta\left(\omega_{0}-\omega\right) \omega ; \quad \phi_{-}(\omega)=\frac{2}{\omega_{0}^{2}} \theta\left(\omega_{0}-\omega\right)\left(\omega_{0}-\omega\right) .
$$

The $\theta$ function guarantees that the maximum value of $\omega$ is $\omega_{0}$. One may find that if taking $\left(\omega_{0}=2 \bar{\Lambda}\right)$, the above solution for the B-meson wavefunction agrees with the result in Ref. 11]. Eq.(14) shows that the dependence on transverse and longitudinal momenta is strongly correlated through the combination $\mathbf{k}_{\perp}^{2} /\left[\omega\left(\omega_{0}-\omega\right)\right]$. Similar transverse momentum behavior has been discussed in Ref. 15] and has been obtained by using the dispersion relations and the quark-hadron duality [16], where they stated that the $k_{T}$ dependence of the wavefunction depends on the off-shell energy of the valence quarks, i.e. $\sim \mathbf{k}_{\perp}^{2} / x(1-x)$, where $x$ is the 
momentum fraction carried by the valence quarks. Under such kind of $k_{T}$-dependence, by doing the Fourier transformation, we find that the correlation between $\omega$ and $z^{2}$ for the transverse part combines in a way like $\left[z^{2} \omega\left(\omega_{0}-\omega\right)\right]$. Thus the above results has been proved completely and Eqs.(45) impose a strong constraint, i.e. $\chi\left(\omega, z^{2}\right)=\chi\left[\omega \cdot\left(\omega_{0}-\omega\right) \cdot z^{2}\right]$, on the B-meson wavefunction.

However, one may ask a question whether the ansatz (8) is too strong. In order to study the B-meson wavefunction more deeply, we assume that the B-meson wavefunction $\Psi_{ \pm}\left(\omega, z^{2}\right)$ can be constructed in a more general way like,

$$
\Psi_{ \pm}\left(\omega, z^{2}\right)=\Phi_{ \pm}(\omega) \cdot\left[\rho\left(z^{2}\right)+\kappa(\omega)\right] \cdot \chi\left[\omega \cdot\left(\omega_{0}-\omega\right) \cdot z^{2}\right]
$$

Note here $\Phi_{ \pm}(\omega)$ has no exact meaning of the distribution amplitude. Substituting the above equation into Eq.(44), one may find that the non-trivial solutions can only be obtained under the following conditions,

$$
\begin{array}{r}
\frac{\rho^{\prime}\left(z^{2}\right)}{\rho\left(z^{2}\right)} z^{2}\left[\Phi_{+}(\omega)-\Phi_{-}(\omega)\right]+\left[\Phi_{+}(\omega)+\omega \Phi_{-}^{\prime}(\omega)\right]=0 \\
\omega \Phi_{-}(\omega) \kappa^{\prime}(\omega)+\left(\Phi_{+}(\omega)+\omega \Phi_{-}^{\prime}(\omega)\right) \kappa(\omega)=0 \\
\Phi_{+}(\omega)-\frac{\omega}{\omega_{0}-\omega} \Phi_{-}(\omega)=0 .
\end{array}
$$

It can be seen that these equations have the following solutions,

$$
\begin{aligned}
\rho\left(z^{2}\right) & =f_{1}\left(z^{2}\right)^{C}, \kappa(\omega)=f_{3} \omega^{-C}\left(\omega_{0}-\omega\right)^{-C} \\
\Phi_{+}(\omega) & =f_{2} \omega^{1+C}\left(\omega_{0}-\omega\right)^{C}, \Phi_{-}(\omega)=f_{2} \omega^{C}\left(\omega_{0}-\omega\right)^{1+C}
\end{aligned}
$$

where $C$ is arbitrary and $f_{i}(i=1,2,3)$ are undetermined parameters that have nothing to do with $z^{2}$ and $\omega$. Substituting these solutions into Eq.(15) and doing the variable transformation $z^{2} \rightarrow x /\left[\omega\left(\omega_{0}-\omega\right)\right]$, we obtain

$$
\left[\omega^{2} g_{1}(x)+\left(8 \omega_{0} \omega-2 \omega_{0}^{2}\right) g_{2}(x)\right]=0
$$

with

$$
\begin{aligned}
g_{1}(x)= & x f_{3}\left(-3 \chi(x)-2(-6+x) \chi^{\prime}(x)+4 x\left(7 \chi^{\prime \prime}(x)+2 x \chi^{(3)}(x)\right)\right)+ \\
& x^{C} f_{1}\left(\left(4 C^{2}+8 C^{3}-3 x-2 C x\right) \chi(x)+2 x((6+4 C(4+3 C)-\right. \\
& \left.\left.x) \chi^{\prime}(x)+2 x\left((7+6 C) \chi^{\prime \prime}(x)+2 x \chi^{(3)}(x)\right)\right)\right), \\
g_{2}(x)= & x f_{3}\left(2 \chi(x)+(-8+x) \chi^{\prime}(x)-4 x\left(4 \chi^{\prime \prime}(x)+x \chi^{(3)}(x)\right)\right)-
\end{aligned}
$$




$$
\begin{aligned}
& x^{C} f_{1}\left(\left(4 C^{2}(1+C)-(2+C) x\right) \chi(x)+x((8+4 C(5+3 C)-\right. \\
& \left.\left.x) \chi^{\prime}(x)+4 x\left((4+3 C) \chi^{\prime \prime}(x)+x \chi^{(3)}(x)\right)\right)\right),
\end{aligned}
$$

where $\chi^{\prime}(x)=\partial \chi(x) / \partial x, \chi^{\prime \prime}(x)=\partial^{2} \chi(x) / \partial^{2} x$ and $\chi^{(3)}(x)=\partial^{3} \chi(x) / \partial^{3} x$. The functions $g_{i}(i=1,2,3)$ must be set to zero so as to ensure that Eq.(22) always be tenable with the variation of $\omega$. And then we obtain that $\chi(x)$ must satisfy the following equation,

$$
\begin{gathered}
4 x^{2}\left[f_{1} x^{C}+f_{3}\right] \chi^{\prime \prime}(x)+\left[8 C f_{1} x^{1+C}+4 f_{1} x^{C+1}-\right. \\
\left.4 f_{3} x\right] \cdot \chi^{\prime}(x)+\left[4 C^{2} f_{1} x^{C}-f_{1} x^{C+1}+f_{3} x\right] \chi(x)=0 .
\end{gathered}
$$

The solution of Eq.(23) from the condition of $f_{3}+f_{1} x^{C}=0$ should be excluded, because when setting $f_{3}+f_{1} x^{C}=0$, one may find that it will lead to $\Psi_{ \pm}\left(\omega, z^{2}\right) \equiv 0$. Under the condition that $f_{3}+f_{1} x^{C} \neq 0$ and by doing the transformation, $\chi(x) \rightarrow \xi(x) /\left(f_{3}+f_{1} x^{C}\right)$, we obtain

$$
4 x \xi^{\prime \prime}(x)+4 \xi^{\prime}(x)-\xi(x)=0
$$

Eq.(24) can be changed into a zero-th normal Bessel function by doing the variable transformation, $x \rightarrow \sqrt{-x}(x<0)$, and then we get $\chi(x)=J_{0}(\sqrt{-x}) /\left(f_{3}+f_{1} x^{C}\right)$.

With the help of the above results, we finally obtain the solution for the B-meson wavefunctions under the ansatz Eq.(16), i.e.

$$
\begin{aligned}
& \tilde{\Psi}_{+}\left(\omega, \mathbf{k}_{\perp}^{2}\right)=\frac{2 \omega}{\pi \omega_{0}^{2}} \theta\left(\omega_{0}-\omega\right) \delta\left(\mathbf{k}_{\perp}^{2}-\omega\left(\omega_{0}-\omega\right)\right) \\
& \tilde{\Psi}_{-}\left(\omega, \mathbf{k}_{\perp}^{2}\right)=\frac{2\left(\omega_{0}-\omega\right)}{\pi \omega_{0}^{2}} \theta\left(\omega_{0}-\omega\right) \delta\left(\mathbf{k}_{\perp}^{2}-\omega\left(\omega_{0}-\omega\right)\right),
\end{aligned}
$$

where the normalization condition, $\int d \omega d^{2} \mathbf{k}_{\perp} \tilde{\Psi}_{ \pm}\left(\omega, \mathbf{k}_{\perp}^{2}\right)=1$, has been adopted.

\section{PHYSICAL CONSEQUENCE AND DISCUSSION}

We have solved the equations which are derived from the equation of motion of the light spectator quark by using two different ansatz, i.e. Eq.(8) and Eq.(16). Under the condition $\omega_{0}=2 \bar{\Lambda}$, the results (see Eqs.(1415) and Eqs.(2526) ) for these two different ansatz agree with the one obtained directly by taking the heavy quark limit $m_{b} \rightarrow \infty[11]$, i.e. these three prescriptions are equivalent. Eqs.(2526) show that the dependence on the transverse and longitudinal momenta is strongly correlated through a non-factorizable combination 


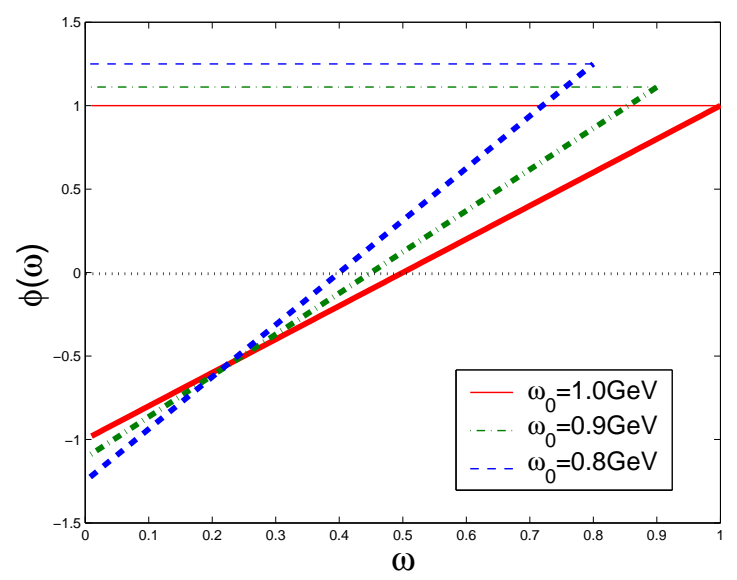

(a)

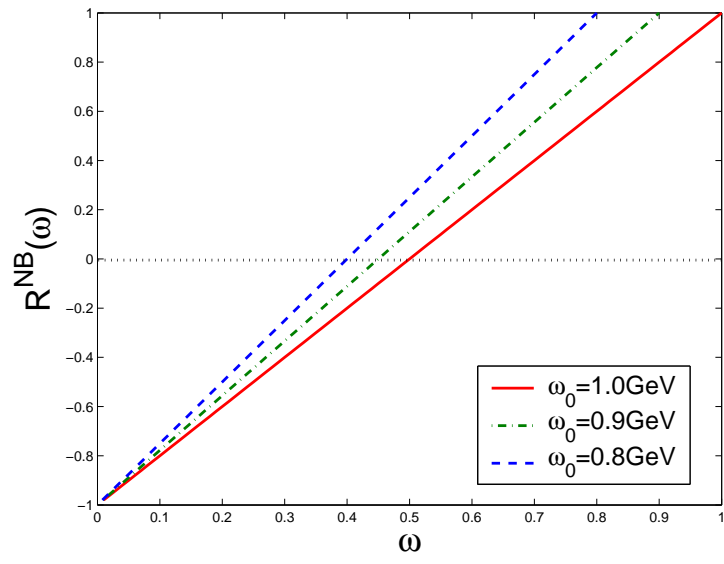

(b)

FIG. 1: The left diagram is the distributions of $\phi_{B}^{N B}(\omega)$ and $\bar{\phi}_{B}^{N B}(\omega)$; the right diagram is the distribution of the ratio $R^{N B}(\omega)=\bar{\phi}_{B}^{N B}(\omega) / \phi_{B}^{N B}(\omega)$. In the left diagram, the thicker same type lines are for $\bar{\phi}_{B}^{N B}(\omega)$ and the thinner ones are for $\phi_{B}^{N B}(\omega)$, respectively.

$\mathbf{k}_{\perp}^{2} /\left[\omega\left(\omega_{0}-\omega\right)\right]$. As has been pointed out in Refs. 11], such kind of transverse momentum dependence for the B-meson wavefunction has a slow-dumping with oscillatory behavior at large transverse distances, $\Psi_{ \pm}\left(\omega, z^{2}\right) \sim \cos \left(\left|\mathbf{z}_{\perp}\right| \sqrt{\omega\left(\omega_{0}-\omega\right)}-\pi / 4\right) / \sqrt{\left|\mathbf{z}_{\perp}\right|}$. This behavior is quite different from that of the models with a simple Gaussian distribution, which has a strong dumping behavior at large transverse distances.

Next, we make a discussion on the behavior of the obtained B-meson distribution amplitudes. For latter convenience, we label the B-meson distribution amplitudes in Eq.(15) as $\phi_{ \pm}^{N B}(\omega)$. The value of $\omega_{0}$ in Eq.(15) can be taken as the continue threshold of the light quarks in the B meson [3, 19], which is usually taken to be in the interval $(0.8-1.0) \mathrm{GeV}^{2}$. Other than taking $\phi_{ \pm}(\omega)$ directly into calculations, one usually takes the combined form of $\phi_{ \pm}(\omega)$, i.e.

$$
\phi_{B}(\omega)=\frac{\phi_{+}(\omega)+\phi_{-}(\omega)}{2}, \quad \bar{\phi}_{B}(\omega)=\frac{\phi_{+}(\omega)-\phi_{-}(\omega)}{2} .
$$

Another typical definition for $\phi_{B}(\omega)$ and $\bar{\phi}_{B}(\omega)$ can be found in Ref. [20], however one may find that the qualitative conclusions are similar. We show the distributions of $\phi_{B}^{N B}(\omega)$ and $\bar{\phi}_{B}^{N B}(\omega)$ with varying $\omega_{0}$ in Fig. (1a) and the distributions of the ratio $R^{N B}(\omega)=$ $\bar{\phi}_{B}^{N B}(\omega) / \phi_{B}^{N B}(\omega)$ in Fig. (11 b). One may observe that the value of $\phi_{B}^{N B}(\omega)$ is always bigger

\footnotetext{
${ }^{2}$ observing that $\bar{\Lambda} \in(0.4,0.5) \mathrm{GeV}, \omega_{0}$ roughly agrees with the relation $\omega_{0}=2 \bar{\Lambda}$.
} 

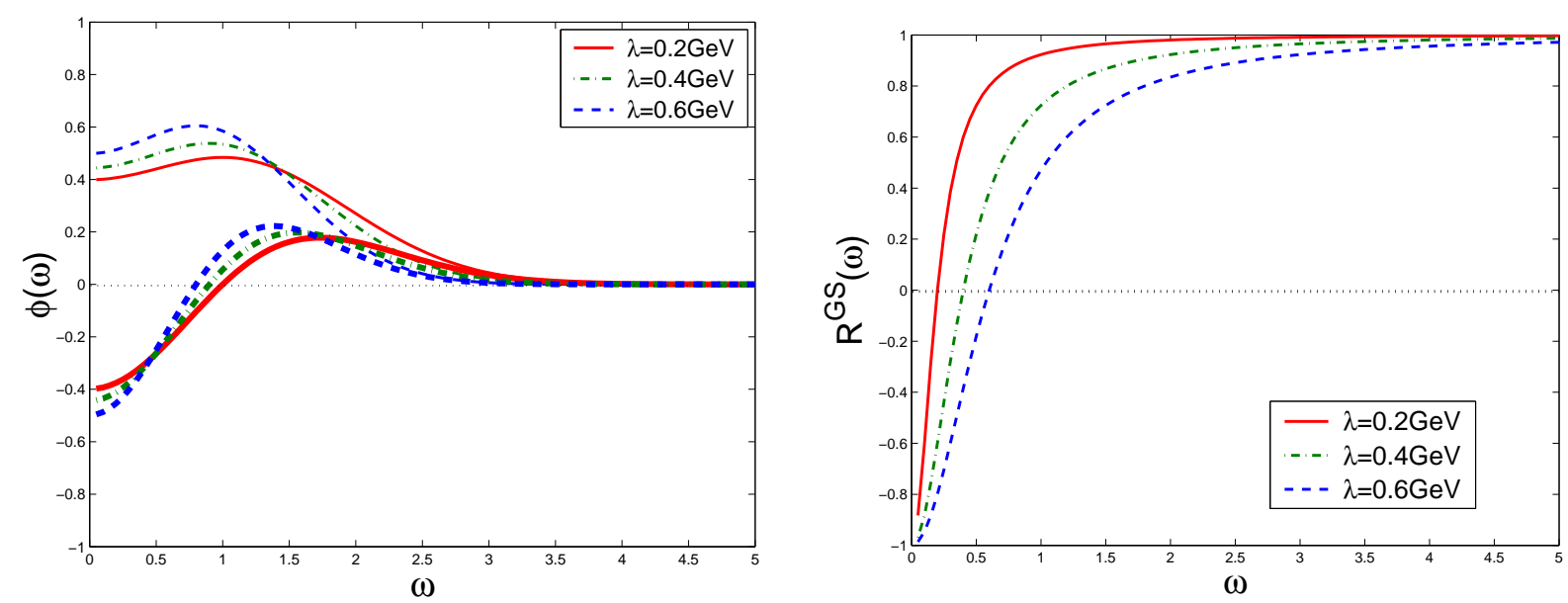

FIG. 2: The left diagram is the distributions of $\phi_{B}^{G S}(\omega)$ and $\bar{\phi}_{B}^{G S}(\omega)$; the right diagram is the distribution of the ratio $R^{G S}(\omega)=\bar{\phi}_{B}^{G S}(\omega) / \phi_{B}^{G S}(\omega)$. In the left diagram, the thicker same type lines are for $\bar{\phi}_{B}^{G S}(\omega)$ and the thinner ones are for $\phi_{B}^{G S}(\omega)$, respectively.

than $\bar{\phi}_{B}^{N B}(\omega)$ and the value of $\bar{\phi}_{B}^{N B}(\omega)$ is negative in small $\omega$ regions.

Some other models for the B meson DAs have also been adopted in the literature. In Ref. 7], the authors adopted the model,

$$
\phi_{+}^{G S}(\omega)=\sqrt{\frac{2}{\pi \lambda^{2}}} \frac{\omega^{2}}{\lambda^{2}} \exp \left(-\frac{\omega^{2}}{2 \lambda^{2}}\right), \phi_{-}^{G S}(\omega)=\sqrt{\frac{2}{\pi \lambda^{2}}} \exp \left(-\frac{\omega^{2}}{2 \lambda^{2}}\right),
$$

which are obtained under the prescription that the transverse part and the longitudinal part of the B wavefunction can be strictly factorized 6 , 7]. In fact, $\phi_{ \pm}^{G S}(\omega)$ can be derived from Eqs.(45) by requiring $\partial \chi\left(\omega, z^{2}\right) / \partial \omega=0$ together with the ansatz Eq.(8). The parameter $\lambda$ can be determined through $\lambda^{2}=4 \partial \chi\left(\omega, z^{2}\right) /\left.\partial z^{2}\right|_{z^{2} \rightarrow 0}$, whose value is of order $\Lambda_{Q C D}[7]$. Inspired by a QCD sum rule analysis, Grozin and Nuebert 17] have proposed a simple model for the distribution amplitudes,

$$
\phi_{+}^{G N}(\omega)=\frac{\omega}{\Omega_{0}^{2}} \exp \left(-\frac{\omega}{\Omega_{0}}\right), \phi_{-}^{G N}(\omega)=\frac{1}{\Omega_{0}} \exp \left(-\frac{\omega}{\Omega_{0}}\right),
$$

where $\Omega_{0}=2 \bar{\Lambda} / 3$. Both $\phi_{ \pm}^{G S}(\omega)$ and $\phi_{ \pm}^{G N}(\omega)$ satisfy the relation Eq.(17). It means that $\phi_{ \pm}^{N B}(\omega), \phi_{ \pm}^{G S}(\omega)$ and $\phi_{ \pm}^{G N}(\omega)$ all are solutions of Eqs.45 as $z^{2} \rightarrow 0 . \phi_{ \pm}^{N B}(\omega)$ and $\phi_{ \pm}^{G N}(\omega)$ have different behaviors, however they have the same asymptotic behavior that is favored by most of the calculations [2, 3, 17, 18], $\phi_{+}^{N B, G N}(\omega) \sim \omega, \phi_{-}^{N B, G N}(\omega) \sim$ const, as $\omega \rightarrow 0$. $\phi_{ \pm}^{G N}(\omega)$ and $\phi_{ \pm}^{G S}(\omega)$ have similar behavior (exponential form), but the asymptotic behavior for $\phi_{+}$is different, i.e. $\phi_{+}^{G S}(\omega) \sim \omega^{2}$, as $\omega \rightarrow 0$. More clearly, we show the distributions 
of $\phi_{B}^{G S}(\omega)$ and $\bar{\phi}_{B}^{G S}(\omega)$, and the ratio $R^{G S}(\omega)=\bar{\phi}_{B}^{G S}(\omega) / \phi_{B}^{G S}(\omega)$ with varying $\lambda$ in Fig.(2). From Fig.(2), one may observe that similar to the case of $\phi_{B}^{N B}(\omega)$ and $\bar{\phi}_{B}^{N B}(\omega), \bar{\phi}_{B}^{G S}(\omega)$ is also comparable to $\phi_{B}^{G S}(\omega)$ in the endpoint regions.

One may observe from Figs.(12) that the value of $\phi_{B}^{N B, G S}(\omega)$ is always bigger than $\bar{\phi}_{B}^{N B, G S}(\omega)$ and the value of $\bar{\phi}_{B}^{N B, G S}(\omega)$ is negative in small $\omega$ regions, such behavior might lead to the total net contribution from $\bar{\phi}_{B}^{N B, G S}(\omega)$ be much smaller than that of $\phi_{B}^{N B, G S}(\omega)$. In literature, many authors (see Refs. 21, 22, 23]) did the phenomenological analysis with a single distribution amplitude $\phi_{B}$, setting $\bar{\phi}_{B}=0$ (or strictly speaking, ignoring the contributions from $\left.\bar{\phi}_{B}\right)$. However, since $\phi_{+}^{N B, G S}(\omega)$ and $\phi_{-}^{N B, G S}(\omega)$ have a quite different endpoint behavior, such difference maybe strongly enhanced by the hard scattering kernel. The results in Refs. 24, 25] for $B \rightarrow \pi$ transition form factor confirm this observation. Especially in Ref. 25], by comparing the PQCD results in the large recoil regions with those obtained from the QCD light-cone sum rules and the extrapolated lattice QCD, the authors give a detailed analysis on the consistent calculation of the $B \rightarrow \pi$ transition form factor in the whole physical region. Their results show that a better slope of the PQCD results can be obtained by taking both $\phi_{B}$ and $\bar{\phi}_{B}$ into consideration. In fact, a discussion on using a single $\phi_{B}$ is given in Ref.[23], which is based on the assumption that $\phi_{-}$vanishes at the both ends of the momentum $\omega$. There is therefore no convincing motivation for setting $\bar{\phi}_{B}=0$ and such an approximation may lead to unreliable results. Since for the endpoint region, the contribution from the hard scattering part might be big, which is the case of $B \rightarrow \pi$ and $B \rightarrow \rho[26]$ transition form factors, so we argue that both $\phi_{B}$ and $\bar{\phi}_{B}$ should be kept for a better understanding of the B physics.

\section{CONCLUSION}

In summary, we have solved the B meson wavefunction based on the equations derived from the vacuum-to-meson matrix element of the nonlocal operators in the heavy quark effective theory and from the equation of motion of the light spectator quark in the B meson. Our analysis shows that the equation of motion of the light spectator quark can impose a strong constraint on the B-meson wavefunctions $\Psi_{ \pm}\left(\omega, z^{2}\right)$. For example, the function $\chi\left(\omega, z^{2}\right)$ depends only on a single combined variable $\left[\omega \cdot\left(\omega_{0}-\omega\right) \cdot z^{2}\right]$, if assuming $\Psi_{ \pm}\left(\omega, z^{2}\right)=\phi_{ \pm}(\omega) \chi\left(\omega, z^{2}\right)$. According to our discussion, the distribution amplitude $\bar{\phi}_{B}(\omega)$ 
of the B meson can not be safely neglected for a better understanding of the B decays.

\section{Acknowledgements}

This work was supported in part by the Natural Science Foundation of China (NSFC).

[1] G.P. Lepage and S.J. Brodsky, Phys.Lett. B87, 359(1979); Phys.Rev. D22, 2157(1980); A.V. Efremov and A.V. Radyushkin, Phys.Lett. B94, 245(1980).

[2] B.O. Lange and M. Neubert, Phys.Rev.Lett. 91, 102001(2003).

[3] V.M. Braun, D.Y. Ivanov and G.P. Korchemsky, Phys.Rev. D69, 034014(2004).

[4] B.O. Lange, Eur.Phys.J. C33, S259(2004).

[5] H. Kawamura, J. Kodaira, C.F. Qiao and K. Tanaka, Phys.Lett. B523, (111)2001, Erratumibid. B536, 344 (2002).

[6] M. Beneke, T. Feldmann, Nucl.Phys. B592, 3(2001).

[7] S.D. Genon and C.T. Sachrajda, Nucl.Phys. B625,239(2002).

[8] H.N. Li and H.S. Liao, Phys.Rev. D70, 074030(2004).

[9] J. Botts and G. Sterman, Nucl.Phys. B225, 62(1989); H.N. Li and G. Sterman, Nucl.Phys. B381, 129(1992); M. Nagashima and H.N. Li, Phys.Rev. D67, 034001(2003), and references therein.

[10] A. Szczepaniak, E.M. Henley and S. Brodsky, Phys.Lett. B243, 287(1990).

[11] H. Kawamura, J. Kodaira, C.F. Qiao and K. Tanaka, Nucl.Phys. B(Proc.Suppl.)116, 269(2003); Mod.Phys.Lett. A18, 799(2003).

[12] N. Isgur and M.B. Wise Phys. Lett. B232, 113 (1989); N. Isgur and M.B. Wise Phys. Lett. B237, 527(1990); E. Eichten and B. Hill, Phys.Lett. B234, 511(1990).

[13] S. Wandzura and F. Wilczek, Phys. Lett. B72, 195(1977).

[14] H. Georgi, Phys.Lett. B240, 447(1990); A.F. Falk, H. Georgi, B. Grinstein and M.B. Wise, Nucl.Phys. B343,1(1990); M. Neubert, Phys. Rept. 245, 259 (1994).

[15] T. Huang, in Proceedings of XXth International Conference on High Energy Physics, Madison, Wisconsin, 1980, edited by L.Durand and L.G. Pondrom, AIP Conf.Proc.No. 69(AIP, New 
York, 1981), p1000; S.J. Brodsky, T. Huang and G.P. Lepage, in Particles and Fields, Vol.2, Proceedings of the Banff Summer Institute, Banff, Alberta, 1981, edited by A.Z. Capri and A.N. Kamal (Plenum, New York, 1983), P143.

[16] I.E. Halperin, A. Zhitnitsky, Phys.Rev. D56, 184(1997).

[17] A.G. Grozin and M. Neubert, Phys.Rev. D55, 272(1997).

[18] V.L. Chernyak and A.R. Zhitnitsky, Phys.Rep. 112, 173(1984).

[19] P. Ball and V.M. Braun, Phys.Rev. D49, 2472(1994); M. Neubert, Phys.Rev. D45, 2451(1992); E. Bagen, P. Ball, V.M. Braun and H.G. Dosch, Phys.Lett. B278, 457(1992); M. Neubert, Phys.Rep.245, 259(1994).

[20] C.D. Lu and M.Z. Yang, Eur.Phys.J. C28, 515(2003).

[21] H.N. Li, Phys.Rev. D52, 3958(1995); H.N. Li and B. Melic, Eur.Phys.J. C11, 695(1999); C.D. Lü, K. Ukai and M. Yang, Phys.Rev. D63, 074009(2001); M. Dahm, R. Jaco and P. Kroll, Z.Phys. C68, 595(1995).

[22] T. Huang and C.W. Luo, Commun.Theor.Phys. 22, 473(1994); A. Szczepaniak, E.M. Henley and S.J. Brodsky, Phys.Lett. B243, 287(1990); S.J. Brodsky, SLAC-PUB-5529; S.J. Brodsky, SLAC-PUB-5917.

[23] Y.Y. Keum, H.N. Li and A.I. Sanda, Phys.Rev. D63, 054008(2001).

[24] Z.T. Wei and M.Z. Yang, Nucl.Phys. B642, 263(2002).

[25] T. Huang and X.G. Wu, hep-ph/0412417

[26] N. Mahajan, hep-ph/0405161 